\title{
The Emergence and Behavioral Stability of Social Status in Green Anole Lizard (Anolis carolinensis) Dyads
}

\author{
William J. Farrell, ${ }^{1, *}$, Devika T. Nair², Brian M. Miller², \& Alan M. Zigler² \\ ${ }^{1}$ Indiana University of Pennsylvania, Indiana, PA \\ ${ }^{2}$ Franklin \& Marshall College, Lancaster, PA \\ *Corresponding author (Email: wfarrell@iup.edu)
}

Citation - Farrell, W. J., Nair, D. T., Miller, B. M., \& Zigler, A. M. (2016). The emergence and behavioral stability of social status in green anole lizard (Anolis carolinensis) dyads. Animal Behavior and Cognition, 3(3), 180-197. doi: $10.12966 / \mathrm{abc} .06 .08 .2016$

\begin{abstract}
Male green anole lizards engage in behavioral displays and stress-hormone mediated color changes during territorial aggression. We examined 12 male dyads during two weeks of cohabitation to document the aggressive behavior of dominant and subordinate animals and to examine the emergence and behavioral stability of dominant/subordinate social status. Two hour observations conducted on alternating days (Days $2-14$ ) of cohabitation indicated that dyads established stable dominate/subordinate relationships in which dominant animals performed more aggressive displays, were typically greener, and monopolized space and resources relative to their subordinate opponent during two weeks of cohabitation. These behavioral asymmetries emerged rapidly following dyad formation; animals that were dominant during prolonged cohabitation exhibited more aggressive displays (pushups and dewlap extensions) and greener body color than subordinates during the first two hours of cohabitation (Day 1). In contrast, display and eyespot blackening latencies did not differ between animals that eventually emerged as dominant and subordinate. These results provide the most in-depth longitudinal description and analysis of stable asymmetries in the display behavior exhibited by male green anoles during long-term cohabitation to date and suggest that anole aggressive displays are honest signals. They also indicate that display intensity/persistence and body color can be reliably used to distinguish dominant from subordinate animals both at the group and dyad levels of analysis during the first two hours of cohabitation.
\end{abstract}

Keywords - Aggression, Agonistic, Social status, Dominant, Subordinate, Green anole, Anolis carolinensis

Most, if not all, social vertebrates exhibit some form of aggressive behavior, and review of the literature indicates that the expression and coordination of aggressive behavior is plastic. Both experience gained during aggressive interactions (Forster, Watt, Korzan, Renner, \& Summers, 2005; Hsu, Earley, \& Wolf, 2006; Yang, Phelps, Crews, \& Wilczynski, 2001) and hormones (de Almeida, Cabral, \& Narvaes, 2015; Haller, 2014; Soma, Scotti, Newman, Charlier, \& Demas, 2008; Wallen, 2005) can modulate aggressive behavior. Additionally, aggressive experience can influence hormone levels (Greenberg \& Crews, 1990; Oyegbile \& Marler, 2005; Wingfield \& Wada, 1989; Wittig, Crockford, Weltring, Deschner, \& Zuberbuhler, 2015; Yang \& Wilczynski, 2003) and central nervous system function (Becker \& Marler, 2015; Fuxjager et al., 2010). In many species including humans (Camras, 1977; Grant, 1969) the outcomes of aggressive interactions are influenced not only by physical combat, but also by aggressive displays, and the outcome of initial encounters can lead to the formation of dominant/subordinate relationships (Riechert, 1998; Ryan \& Wilczynski, 2011). A thorough understanding of aggressive behavior involves, in part, understanding how experience gained during initial aggressive interactions (including displays) can alter the behavior of opponents and the dynamics 
of the long-term social interactions that follow.

Green anole lizards (Anolis carolinensis) are frequently used as a neuroethological model organism for the study of aggressive behavior and social status. During the breeding season (April July), male green anoles engage in aggressive interactions with male conspecifics to establish territories that contain multiple females and exclude other males (Jenssen \& Nunez, 1998; Ruby, 1984). Although data indicating the amount of time spent defending and establishing breeding territories are sparse, the results of one field study indicate that approximately $10 \%$ of a breeding male's time-budget is used for territorial defense (Jenssen, Greenberg, \& Hovde, 1995).

Male green anole aggressive interactions are characterized by both species-typical displays and by hormonally regulated changes in skin color (Greenberg \& Crews, 1983). Aggressive, behavioral displays in this species typically include pushups (PU), extensions of the red dewlap (DE), or throat fan, and lateral compression (LC) of the body along the sagittal axis. PU are effected by flexion and extension of the forelimbs, and can occur with or without concurrent head nods, creating a distinct up-and-down bobbing motion. DE and PU are common to all phases of aggressive interaction. If both animals continue to display, and the aggressive interaction intensifies, one or both animals will also become laterally compressed and continue to produce PU and/or DE while circling their opponent and exposing their enhanced vertical profile (Greenberg \& Crews, 1983). Skin color also changes during aggressive interactions. Body color can vary from bright green to dark brown, and a patch of skin behind the eye, referred to as the postorbital eyespot, blackens during heightened aggressive interactions (Greenberg \& Crews, 1983).

Green anoles have become a popular model organism for studying the neuroendocrinology of aggression and social status in part because male anoles readily display species-typical aggressive behavior in the laboratory, and these behaviors can be quantified. In addition, skin color changes during aggressive interactions can be used to estimate adrenal stress hormone levels. Increased levels of circulating catecholamines blacken the postorbital eyespot (Goldman \& Hadley, 1969). Browning of the body skin, which is influenced by melanocyte-stimulating hormone (MSH) and catecholamines (Goldman \& Hadley, 1970; Vaughan \& Greenberg, 1987), is sometimes accompanied by increased plasma titers of corticosterone and can indicate a state of stress or social subordination (Greenberg, Chen, \& Crews, 1984; Greenberg \& Crews, 1990). Importantly, the neural and endocrine substrates involved in the regulation of aggressive behavior appear to be largely conserved across vertebrate taxa, enhancing the external validity of the model (Korzan \& Summers, 2007; Summers, 2001; Summers \& Winberg, 2006).

The popularity of green anoles as a model organism for the study of social status (dominant/subordinate) also rests on findings indicating that the outcome of dyadic aggressive interactions can be predicted quickly and that the winner of an initial aggressive interaction reliably achieves stable, long-term, dominant status (e.g., Greenberg \& Crews, 1990; Plavicki, Yang, \& Wilczynski, 2004; Summers \& Greenberg, 1995). These characteristics can facilitate the examination of individual differences related to the establishment of social status because dominant and subordinate animals can quickly and reliably be identified without the need for, or potentially confounding effects of, prolonged aggressive interaction.

Whereas social status following aggressive interactions is typically described as stable, with dominant animals having disproportionate access to desirable perching sites and resources (e.g., food and mates), close inspection of the literature suggests the possibility of a more dynamic picture. For example, Greenberg et al. (1984) indicate that weeks may be required for stable dominant/subordinate relationships to emerge. Comprehensive, quantitative, longitudinal data documenting the aggressive behavior of dominant and subordinate animals during prolonged cohabitation are lacking in the literature. Such data would be useful for understanding the emergence and dynamics of social status relationships and for assessing the stability of these relationships over time.

Longitudinal data collected during long-term cohabitation that support the assertion of stable dominant/subordinate relationships are typically limited to measures of perch site selection and body color (e.g., Plavicki et al., 2004). Quantitative data documenting the aggressive behavior of dyad members are likely excluded, in part, because the frequencies of these behaviors decline dramatically 
after the initial interaction, and researchers typically assess dominant/subordinate social status using brief observations that preclude capturing representative samples of display behavior. In the present study we used digital video recording (rather than brief, live observations) to gather quantitative, time-course data on the aggressive displays exhibited by dominant and subordinate male green anoles during long-term cohabitation. We tested the hypothesis that the dyad members operationally defined as dominant consistently exhibit more of these displays than their subordinate counterparts on Days $2-14$ of cohabitation. We also determined whether the display asymmetries between dominant and subordinate animals that characterize long-term cohabitation reliably emerged during the first two hours of cohabitation (Day 1).

In addition, because it is often desirable to identify dominant and subordinate individuals in a dyad quickly, without requiring prolonged cohabitation, we attempted to identify early indicators of social status. We examined the extent to which measures of behavioral/physiological reactivity (latencies to exhibit aggressive displays and begin blackening the post-orbital eyespot) and display intensity/persistence (display frequency and duration) obtained during the initial two hours of cohabitation were reliable, early indicators of eventual dominant/subordinate, social status because differences in one or both of these dimensions distinguish winners from the losers of aggressive contests across multiple taxa (Hsu et al., 2006). In green anoles numerous reports indicate that males with shorter latencies to initiate blackening of the postorbital eyespot (e.g., Larson \& Summers, 2001; Plavicki et al., 2004; Summers \& Greenberg, 1994) and engage in other aggressive displays (Korzan, Øverli, \& Summers, 2006) reliably win aggressive contests while others (Wilczynski, Salem, Ezeoke, \& Black, 2015; Zaman \& Farrell, 2007) have found measures of display intensity/persistence to be more robust predictors of winning or losing. For this study, we hypothesized that the animals that eventually became dominant would respond to their opponents more quickly (heightened behavioral/physiological reactivity) and display more (increased display intensity/persistence) than their opponent during the first two hours of cohabitation.

To accomplish these goals, we used digital video recording to document (two hours per day) the behavioral interactions amongst pairs of male anoles during two weeks of cohabitation. Dyad members were classified as dominant or subordinate based on their behavior during a $30 \mathrm{~min}$ courting test (female introduced into enclosure) on Day 2. Video records from the first two hours of cohabitation (Day 1) were used to examine whether behavioral differences between dominant and subordinate animals emerged during the first two hours of cohabitation and to assess the extent to which measures of behavioral/physiological reactivity (behavioral and eyespot blackening latencies), measures of display intensity/persistence (PU and DE frequency and DE duration), and body color correctly distinguished the animals that eventually emerged as dominant from those that became subordinate. Recordings made on alternate days from Days $2-14$ (without a female present) were used to determine whether dominant animals exhibited more aggressive displays than subordinates and examine the extent to which the behavioral asymmetries that emerged by Day 2 persisted in a stable fashion during long-term cohabitation. Because green anole social status is frequently used as a dependent measure or as a quasiindependent variable across which comparisons are made, data are reported and analyzed at both the group (dominant/subordinate) and individual dyad levels of analysis.

\section{Method}

\section{Animals}

Wild-caught, sexually mature, male Anolis carolinensis ( $\geq 60 \mathrm{~mm}$ snout-to-vent length: SVL) were purchased from a commercial supplier (Charles Sullivan, Inc., Nashville, TN). Prior to inclusion in the study each male was housed with two females in a 10-gal glass terrarium $(58 \times 28 \times 25 \mathrm{~cm})$. Each enclosure was outfitted with a ribbed plastic perch (1/4" dia.) placed diagonally from the rear, bottom corner of the cage to the top, front corner of the cage and a plastic shelter constructed from a snack-sized, GladWare-type container $(12 \times 9 \times 5 \mathrm{~cm})$ with a small door $(\approx 3 \times 2 \mathrm{~cm})$ cut to grant access. The floor 
was covered with sphagnum peat moss substrate $(\approx 2 \mathrm{~cm}$ deep $)$. Opaque plastic panels were inserted between adjacent terraria to visually isolate inhabitants from their neighbors. Because male green anole aggressive behavior typically occurs during the breeding season and is influenced by testosterone (Crews, Traina, Wetzel, \& Muller, 1978), temperature (32 $\mathrm{C}$ day $/ 23^{\circ} \mathrm{C}$ night), and photoperiod (14 hrs light/10 hrs dark) were set at levels known to maintain reproductive condition (Licht, 1971). Humidity inside the enclosures was $\approx 50 \%$. Illumination was provided by one standard fluorescent bulb and one fluorescent, ultraviolet bulb (Reptisun 10.0, Zoo Med Laboratories, San Luis Obispo, CA) suspended above the screen covering each cage. Additionally, a reflective lamp with a $40 \mathrm{~W}$ incandescent bulb was placed atop each cage directly above the top of the perch to provide additional light and a thermal gradient within the enclosure. An automated misting system was used to elevate the humidity levels inside the enclosures and provided drinking water periodically throughout the day. Crickets or mealworms $(2-4$ per animal) were provided as prey items three times a week. Male anoles were screened for reproductive condition by introducing a novel female into their home enclosure. Only the males who responded with courting behavior (Crews, 1975) were included in the study. Twelve pairs of male anoles (24 animals) were used in the study. All housing and research procedures were approved by the Franklin \& Marshall College Institutional Animal Care and Use Committee.

\section{Procedure}

At the beginning of the study, sexually responsive males were size-matched (by SVL) with an available opponent and placed individually into opposing sides of a 10-gal terrarium separated by an opaque, removable barrier. For the 12 pairs of anoles used in the study, the mean weight difference $( \pm$ SE) between dyad members was $0.13 \pm 0.05 \mathrm{~g}$ and the mean SVL difference was $2.42 \pm 0.63 \mathrm{~mm}$. Females were not transferred to the testing chambers, which were outfitted in a manner otherwise similar to the terraria used for general housing. A small, green line was drawn on either the left or right side of the base of each lizard's tail to assist with identification of individual animals once dyadic interactions began. Marks were checked on a daily basis throughout the study (after any observations were performed for that day), and if an animal had shed, or a mark had faded, it was refreshed. Body color was also recorded one time on each day prior to cohabitation to obtain baseline values.

After five days in the divided terrarium, the wall separating adjacent males was removed, a single perch was placed in the enclosure, and behavior was video-recorded for two hours. A trained observer was stationed in the testing room during this initial observation to ensure that the latency to eyespot blackening was accurately recorded. Following this initial observation (Day 1), dyads were left intact and similar, two-hour video records were obtained on alternating days from Day 2 through Day 14 of cohabitation. Following the completion of each of these observations, a single female anole was placed in each enclosure for $30 \mathrm{~min}$. Video records of these "courting tests" were used to categorize animals as dominant and subordinate (Day 2 only) and confirm that social status did not change during the remainder of the study. All behavioral observations were conducted between 9:00 AM and 5:00 PM (lights on = 7:00 AM), and, to the extent possible, dyads were observed at the same time each day.

Observer effects were minimized by keeping the main room lights off (cages were illuminated) and by monitoring/recording the majority of observations from an adjacent procedure room. Video records were generated using color, pan-tilt-zoom, network cameras (Panasonic, WV-SC385) connected to an Apple Macintosh computer in the procedure room. SecuritySpy software (bensoftware.com) was used to record observations and control the pan, tilt, and zoom functions of the cameras. Custom software was used to manually set the focus and exposure levels of the cameras in order to address occasional errors by the auto-focus system and keep exposure levels consistent during video recording. Disturbances by animal care staff and researchers were minimized throughout the study, and routine colony procedures (e.g., feeding and filling the misting system) were performed after observations were completed for the day. Cages were not cleaned during the study. 


\section{Behavioral Quantification}

Behavior was coded using Apple Macintosh computers and EventCoder software (Goldstein \& Brodsky, 2006). This software allows the user to assign behaviors to specific keys on the computer keyboard and depress keys during occurrences of the specified behaviors. Each depression of a key is registered as a behavioral occurrence, and the duration of key depression can be recorded as the duration of that occurrence. Importantly, this software uses the millisecond time-code inherent in digital video files allowing the user to speed-up, slow-down, reverse or pause video playback while still generating an accurate behavioral record. The individuals coding video records from the first day of cohabitation (the observation used to predict eventual social status) were blind to which dyad members appeared to be dominant on Days $2-14$ and vice versa. Social status was not determined until all behavioral coding was complete.

The behaviors coded during the study are listed in Table 1 along with their definitions, abbreviations, the types of measurements obtained on various days (e.g., latency, frequency, duration), and inter-rater reliability values. For the initial (Day 1), two-hour observation of the dyads immediately following wall removal, the behaviors coded were the frequency of PU and DE, the duration of DE and the latency to initiate eyespot blackening, LC, PU and DE. Behaviors were coded separately for each member of the dyad. A single PU was registered for each upward movement of the head/torso and a single DE was registered for each visible extension and complete retraction of the dewlap. Body color was also coded every minute on a scale of 1 to $3(1=$ completely green, $2=$ mix of brown and green, $3=$ completely brown).

For the two-hour observations conducted on alternating days from Day 2 through Day 14 of cohabitation, the behaviors coded were the frequency of PU and DE, the duration of DE, the frequency with which each animal displaced their opponent from an occupied location within the enclosure (displacements) and the number of times they chased their opponent (chases). Displacements and chases were added to the coding scheme for these observations to assess the extent to which dominant animals monopolized space. Each animal's body color was also rated at the beginning and end of these observations, and the mean of the two ratings was used as a single, daily, body color score.

Behavior during the courting test on Day 2 of cohabitation was coded to categorize animals as dominant or subordinate. We quantified the frequency of four different displays associated with courting (Crews, 1975; Greenberg \& Crews, 1983): PU, DE, rapid nods and strutting (see Table 1 for definitions). Counts of PU and DE were registered as discussed above, and single occurrences of rapid nods and strutting were registered for each behavioral bout that occurred without a discernable pause. Male-male chases and displacements were also coded. Behavior was not quantified during the courting tests conducted on later days, but the video records were reviewed to assure that social status did not change. Due to malfunction of the video recording system, video records of a single two-hour observation on Day 10 of cohabitation and a single courting test on Day 14 of cohabitation were lost. Body color measurements from the beginning and end of the two-hour observation on Day 10 were manually obtained and were therefore not lost.

\section{Data Analysis}

The dyad member that exhibited the highest combined frequency of displays (PU, DE, rapid nod and strutting combined) during the courting test on Day 2 of cohabitation was classified as the dominant animal, and the remaining animal was categorized as subordinate. Because we wished to categorize animals as dominant or subordinate primarily based on courting behavior, we did not count PU and DE that occurred during the $5 \mathrm{~s}$ preceding or following a chase or displacement by either animal or a PU or DE by the opposing dyad member. These displays were excluded because PU and DE occur both during green anole aggressive interactions and courting, and displays that occurred in these contexts often appeared to be aggressive rather than courting-related. 
To determine whether behavioral asymmetries between dominant and subordinate dyad members emerged during the first two hours of cohabitation, and to test the hypothesis that measures of display intensity/persistence (PU and DE frequency and DE duration) are effective early indicators of social status, we compared the total counts of PU and DE and the total DE duration from the first two hours of interaction (Day 1) for dominant and subordinate animals. A similar comparison was made using the mean body color ratings of each animal across the entire two-hour observation to determine whether the animals that eventually emerged as dominant were greener than subordinates. T-tests for correlated samples were used for these comparisons because the behavior of interacting dyad members cannot be considered independent. Parametric tests were chosen for all of these analyses despite the fact that the distribution of difference scores for PU differed from normal (Shapiro Wilk test, $p=0.022$ ). This deviation from normality was attributable to a single outlying data point obtained from the one dyad in which the animal that eventually emerged as dominant produced fewer PU than the animal that became subordinate (with outlier excluded: Shapiro Wilk test, $p=0.510$ ), thereby biasing the test against yielding a significant difference. Sign tests (exact binomial) were also performed on the total counts of PU and $\mathrm{DE}$, the total DE duration, and the mean body color data from the first two-hours of cohabitation to determine whether the proportion of dominant animals exhibiting more displays and a greener average body color differed significantly from the proportion of 0.5 expected by chance. Sign-tests were performed only on the data obtained from the entire, two-hour observation. Additional proportions (for the first 30,60 and $90 \mathrm{~min}$ of interaction) are reported in the results for descriptive purposes.

To account for inflated family-wise error rates, the $\alpha$ level for the three display intensity/persistence comparisons (PU frequency, DE frequency and DE duration) was adjusted to 0.017 (two-tailed) using the Bonferroni correction. For the rare instances $(<1 \%$ of minute to minute observations) where an individual anole's body skin was not visible in the video image for a particular minute of the two-hour interaction on Day 1, the mean of the body color rating values from the preceding and following successful observations were entered into the vacant cells as an estimate of body color.

To test the hypothesis that heightened behavioral/physiological reactivity (responding more quickly) at the beginning of cohabitation is a reliable indicator of future social status, we compared the PU, DE, LC and eyespot blackening latencies from Day 1 across dominant and subordinate dyad members. The Wilcoxon matched-pairs signed ranks test was chosen for these comparisons because the distribution of difference scores between dominant and subordinate dyad members all deviated significantly from normal, and there were multiple outliers for the majority of these comparisons. Sign tests (exact binomial) were also performed on these latency data to determine whether the proportion of dominant animals exhibiting shorter display and eyespot blackening latencies differed significantly from the proportion of 0.5 expected by chance. To account for inflated family-wise error rates, the $\alpha$ level for the four latency comparisons (eyespot blackening, PU, DE and LC) was adjusted to 0.013 (two-tailed) using the Bonferroni correction. For all latency measures, animals that did not express the behavior were assigned the maximum possible latency (7200 s).

To test the hypothesis that animals classified as dominant on Day 2 of cohabitation would display more than subordinates during the two-hour observations conducted on alternating days from Days $2-14$ of cohabitation, separate correlated samples t-tests were performed for PU frequency, DE frequency and DE duration. To perform each t-test, single values were obtained for each dominant and subordinate animal by averaging their behavioral counts or durations for the seven, two-hour observations from Days $2-14$. The t-tests were then used to compare these "mean values per observation" between dominant and subordinate animals. Similar comparisons were performed on body color scores and the frequency of combined chases and displacements to determine whether dominant animals were greener and monopolized space relative to their subordinate counterparts. One-way, repeated measures ANOVAs were used to assess the stability of these behavioral and color asymmetries from Day $2-14$ of cohabitation. These ANOVAs were conducted on difference scores obtained by subtracting the behavioral counts, durations (DE only), and color scores, exhibited by the subordinate animal in each dyad from the equivalent value for the dominant animal for each two-hour observation conducted on alternating days from Days 2 through 14. This yielded a total of 84 cells (12 dyads X 7 observations) for each ANOVA. 
The values in each cell represented the degree of asymmetry between the dominant and subordinate animal for each measure. Due to video equipment malfunction, the video observation from a single dyad was lost on Day 10 of cohabitation. For the purposes of these one-way, repeated measures ANOVAs, the single open cell was filled by averaging the values from the observations conducted on Days 8 and 12 for that dyad.

\section{Table 1}

\section{Aggressive and Courting Behaviors Coded}

\begin{tabular}{|c|c|c|c|c|c|}
\hline \multirow{2}{*}{ Behavior } & \multirow{2}{*}{ Abbrev. } & \multirow{2}{*}{ Definition } & \multicolumn{2}{|c|}{ Measurements Made } & \multirow[t]{2}{*}{ Reliability $^{\mathrm{a}}$} \\
\hline & & & Day 1 & Days $2-14$ & \\
\hline \multicolumn{6}{|l|}{ Aggressive } \\
\hline Pushup & PU & $\begin{array}{l}\text { Upward motion of head/body effected by } \\
\text { the forelimbs }\end{array}$ & $\begin{array}{l}\text { Latency } \\
\text { Frequency }\end{array}$ & Frequency & $r(20)=0.99$ \\
\hline $\begin{array}{l}\text { Dewlap } \\
\text { Extension }\end{array}$ & $\mathrm{DE}$ & $\begin{array}{l}\text { Extension of the dewlap beneath the } \\
\text { throat }\end{array}$ & $\begin{array}{l}\text { Latency } \\
\text { Frequency } \\
\text { Duration }\end{array}$ & $\begin{array}{l}\text { Frequency } \\
\text { Duration }\end{array}$ & $\begin{array}{l}r_{(20)}=0.98 \\
r_{(20)}=0.96\end{array}$ \\
\hline $\begin{array}{l}\text { Lateral } \\
\text { Compression }\end{array}$ & $\mathrm{LC}$ & $\begin{array}{l}\text { Compression of the thorax, yielding a } \\
\text { heightened sagittal profile }\end{array}$ & Latency & NQ & $r_{(20)}=0.99$ \\
\hline Displacement & None & $\begin{array}{l}\text { Displaced animal vacates a position held } \\
\text { for } \geq 30 \mathrm{~s} \text {, following an approach or } \\
\text { display by the displacing animal }\end{array}$ & NQ & Frequency & $r_{(24)}=0.99$ \\
\hline Chase & None & $\begin{array}{l}\text { Chased animal vacates a position held for } \\
<30 \mathrm{~s} \text {, following the approach of chasing } \\
\text { animal }\end{array}$ & NQ & Frequency & $r_{(24)}=0.98$ \\
\hline Eyespot & None & Blackening of skin behind the eye & Latency & NQ & $\mathrm{NA}^{\mathrm{b}}$ \\
\hline Body Color & None & $\begin{array}{l}\text { Color of body skin coded on a three point } \\
\text { scale: } 1=\text { green, } 2=\text { green and brown, } 3 \\
=\text { brown }\end{array}$ & $\begin{array}{l}\text { Coded once } \\
\text { per minute }\end{array}$ & $\begin{array}{l}\text { Coded at } \\
\text { beginning and end } \\
\text { of session }\end{array}$ & $r_{s(40)}=0.96$ \\
\hline \multicolumn{6}{|l|}{ Courting $^{\mathrm{c}}$} \\
\hline Pushup & PU & See above & NA & Frequency & $r_{(24)}=0.99$ \\
\hline $\begin{array}{l}\text { Dewlap } \\
\text { Extension }\end{array}$ & $\mathrm{DE}$ & See above & NA & Frequency & $r_{(24)}=0.99$ \\
\hline Rapid Nod & None & $\begin{array}{l}\text { Rapid vertical movements of the head } \\
\text { effected by the neck musculature }\end{array}$ & NA & Frequency & $r_{(24)}=0.97$ \\
\hline Strutting & None & $\begin{array}{l}\text { Male approaches female with prance-like } \\
\text { gait }\end{array}$ & NA & Frequency & $r(24)=0.99$ \\
\hline
\end{tabular}

Note. Abbrev. = Abbreviation, $\mathrm{NQ}=$ Not Quantified, NA = Not Applicable

${ }^{a}$ Inter-rater correlations calculated using the number of observations in parentheses.

${ }^{b}$ Eyespot latency was coded by a single observer in the room. A second, remote, video observer confirmed the order of eyespot blackening for each dyad.

${ }^{\mathrm{c}}$ The frequency of the four courting behaviors were only coded on Day 2, and they were combined to determine social status. 
Farrell et al. 187

\section{Results}

\section{Determination of Social Status}

The dyad member who exhibited a higher combined frequency of PU, DE, rapid nodding and strutting during the 30 min courting test conducted on Day 2 of cohabitation was classified as dominant and the other dyad member was classified as subordinate. Dominant animals exhibited a mean of 179.67 $(S E=38.81)$ displays during the courting test, while subordinate animals displayed an average of 16.75 $(S E=6.20)$ times. Four of the 12 subordinate animals did not perform displays during the courting test. For the eight dyads in which both the dominant and subordinate animals displayed, the dominant male exhibited a minimum of 2.1 times as many displays as the subordinate. In addition, 11 of the 12 animals classified as dominant exhibited rapid nods and/or strutting, behaviors unique to escalating courting (Crews, 1975). Only five subordinate animals exhibited rapid nods or strutting and they never performed as many of these displays (combined) as their opponent.

\section{Emergence of Behavioral Asymmetries and Early Indicators of Social Status}

Asymmetries in display behavior reliably emerged during the first two hours of dyadic interaction. The mean frequency of PU and DE and the duration of DE during successive 10-min intervals of the first two hours of cohabitation are depicted in Figures $1 \mathrm{a}, \mathrm{b}$, and $\mathrm{c}$ respectively. These figures reveal that, while the animals that eventually became dominant and subordinate displayed with similar intensity shortly after wall removal, the behavior of the groups diverged over time with dominant animals showing more persistence than their subordinate counterparts.

Measures of display intensity/persistence (PU and DE frequency and DE duration) during the first two hours of cohabitation were reliable indicators of future social status (Figure 2a). Animals that eventually became dominant exhibited a significantly higher frequency of PU than subordinates $(t(11)=$ $3.09, p=0.010,95 \%$ CI $[77.11,458.72], d=0.89)$, and a similar difference was observed for DE frequency $(t(11)=3.24, p=0.008,95 \%$ CI $[9.63,50.37], d=0.94)$. DE duration (not depicted) was also significantly greater $(t(11)=4.24, p=0.001,95 \%$ CI [33.96, 107.43], $d=1.22)$ for dominants $(M=89.32$ $\mathrm{s}, S E=11.60 \mathrm{~s})$ compared to subordinates $(M=18.62 \mathrm{~s}, S E=8.64 \mathrm{~s})$.

Individual measures of display intensity/persistence during the initial two hours of interaction also distinguished future dominants from subordinates within individual dyads. Whereas a higher frequency of PU or DE, or a longer duration of DE during the first two hours of cohabitation failed to correctly distinguish all future dominant animals from their subordinates, dominant dyad members were higher on each of these measures than their matched subordinate in 11 of 12 dyads (sign test, exact binomial, $p=0.006$, two-tailed for each behavior). DE frequency and duration remained equally effective as indicators of dominance ( 11 of 12 dyads) when only the first $90 \mathrm{~min}$ of cohabitation were considered, and their effectiveness declined at shorter interaction lengths. Animals that eventually became dominant exhibited more DE than their opponent in 8 of 12 dyads during the first $60 \mathrm{~min}$ of cohabitation and 7 of 12 dyads during the first 30 min of cohabitation. Dominant animals had a longer duration of DE than their opponent in 10 of 12 dyads during the first 60 min of cohabitation and 7 of 12 dyads during the first 30 min of cohabitation. PU frequency was higher for future dominants in 10 of 12 dyads after 90 or 60 min of cohabitation and higher in 9 of 12 dyads after 30 min of cohabitation.

The mean, baseline, body color rating over the four days prior to cohabitation did not differ significantly between dominant $(M=1.68, S E=0.13)$ and subordinate $(M=1.75, S E=0.14)$ animals $(t(11)=0.29, p=0.780)$. Color ratings diverged during the first two hours of cohabitation (Figure 1d) with subordinate animals becoming browner than dominants, and mean body color during the first two hours of cohabitation (Figure $2 \mathrm{~b}$ ) was a reliable predictor of future social status. The mean body color rating of dominant animals was significantly lower (greener) than their subordinate counterparts during the first two hours of cohabitation $(t(11)=4.68, p=0.001,95 \%$ CI $[-1.36,-0.49], d=1.35)$. A lower mean body color rating also distinguished the future dominant animal from the subordinate in 10 of 12 
dyads (sign test, exact binomial, $p=0.039$, two-tailed) after 120, 90 and $60 \mathrm{~min}$, and 9 of 12 dyads after $30 \mathrm{~min}$.

Behavioral/physiological reactivity on the first day of cohabitation was a poor indicator of eventual social status. The mean latencies to initiate eyespot blackening and exhibit PU, DE and LC following removal of the dividing wall on Day 1 of cohabitation are depicted in Figure 3a. Although examination of these data suggests that dominants exhibit shorter latencies for PU, DE and LC, the mean values are heavily influenced by animals that did not engage in various behaviors and therefore had the maximum value $(7200 \mathrm{~s})$ registered as their latency. When the means for dominants and subordinates are recalculated excluding animals that did not display various behaviors or eyespot blackening (Figure $3 \mathrm{~b}$ ), differences across social status are reduced. The results of Wilcoxon Signed-Rank Tests conducted on the complete data sets (including the animals that did not display various behaviors or eyespot blackening) indicated that future dominants and subordinates did not have significantly different eyespot-blackening latencies $(Z=0.63, p=0.569)$ or significantly different latencies to perform $\mathrm{PU}(Z=0.31, p=0.791), \mathrm{DE}$ $(Z=1.33, p=0.204)$ or $\operatorname{LC}(Z=1.80, p=0.077)$.

A

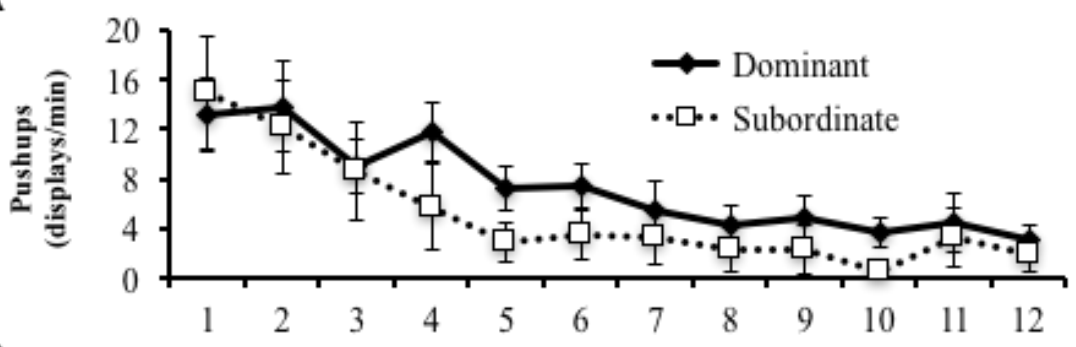

B

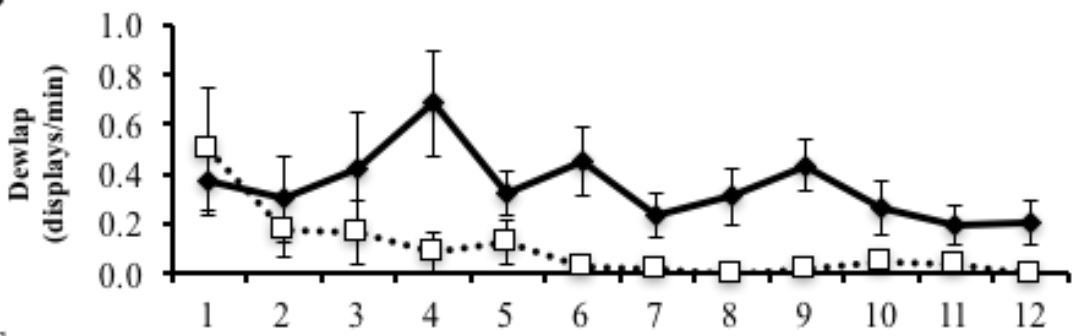

C

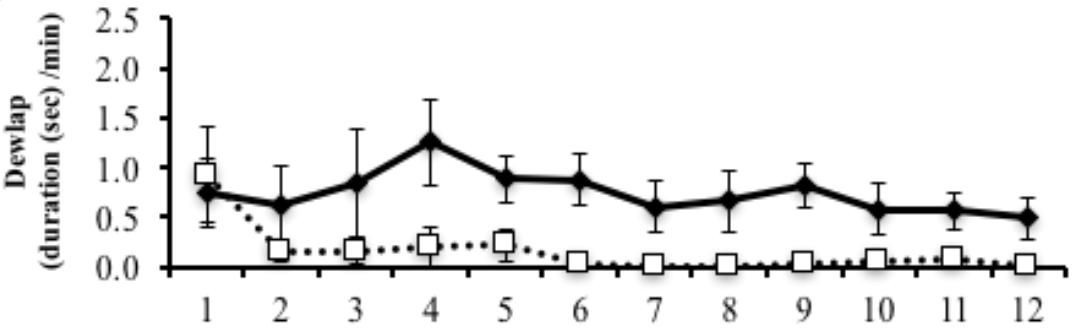

D

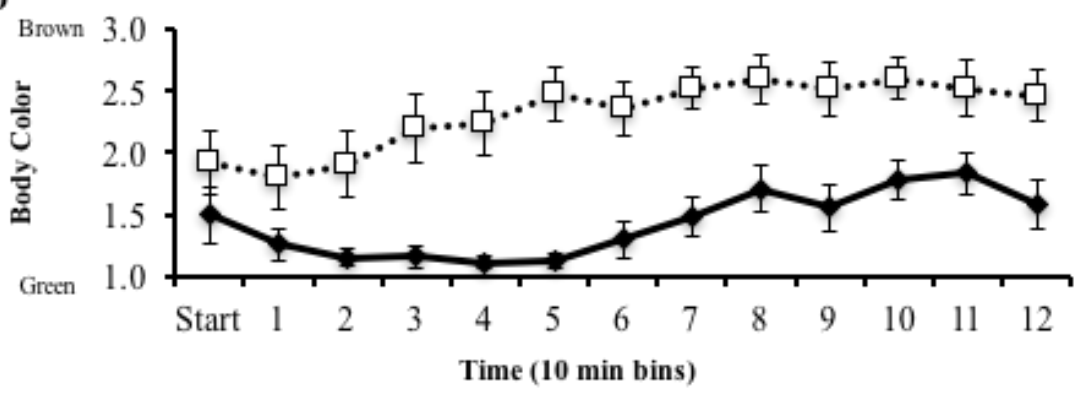

Figure 1. Frequency of Pushups (A) and Dewlap Extensions (B), duration of Dewlap Extension (C), and mean Body Color (D) exhibited by future dominant and subordinate male green anoles during the first 120 minutes of cohabitation. Data are presented as means $\pm S E$. 
The latencies to initiate eyespot blackening and exhibit PU, DE and LC on Day 1 of cohabitation also failed to reliably distinguish between the animals that eventually emerged as dominant and subordinate in individual dyads. Seven future dominants and five future subordinates (7D:5S) exhibited the shorter latency to begin eyespot blackening for their dyad (sign test, exact binomial, $p=0.774$, twotailed), and the proportions were similar for the latencies to perform PU (6D:6S; sign test, exact binomial, $p=1.00$, two-tailed) and DE (7D:5S; sign test, exact binomial $p=0.774$, two tailed). LC latency was the best predictor of future social status with 8 of 12 dominant males displaying a shorter latency than their opponent. The probability of obtaining a proportion this extreme or more extreme, however, still failed to reach statistical significance (sign test, exact binomial, $p=0.388$, two-tailed).

A

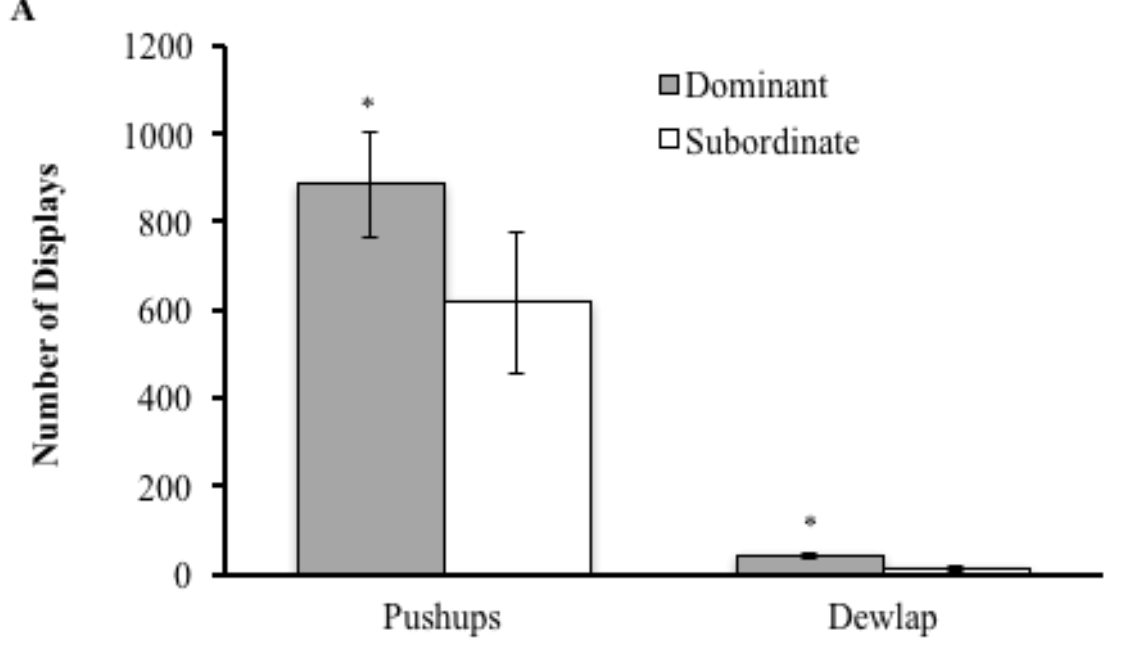

B

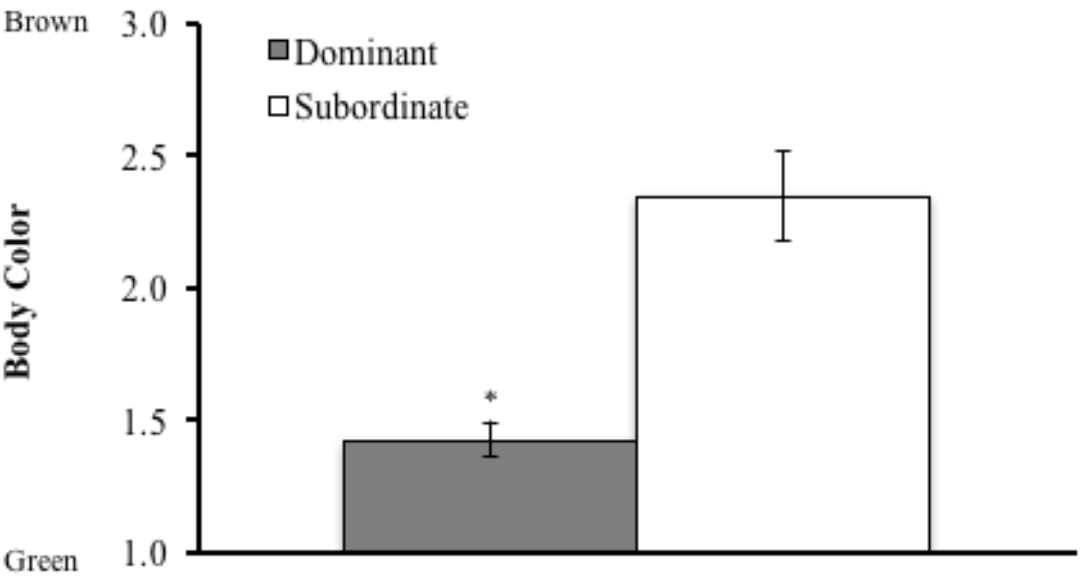

Figure 2. (A) Mean number of pushups and dewlap extensions, and (B) mean body color rating exhibited by dominant and subordinate animals during the first two hours of cohabitation. Data are presented as means $\pm S E$. Asterisks indicate significant differences.

\section{Behavioral Asymmetries from Day 2 through Day 14 of Cohabitation}

The dyad members operationally defined as dominant on Day 2 of cohabitation consistently exhibited more aggressive displays than their subordinate counterparts during prolonged cohabitation. The mean frequencies of PU and DE during the two-hr observations conducted on alternating days from Days 2 - 14 of cohabitation are depicted in Figures 4a and 4b. Animals classified as dominant always displayed more PU than their subordinate opponent during individual two-hour observations. Dominant 
animals also typically (five exceptions in 83 observations) displayed more DE than their opponents. Ttests for correlated samples conducted on the mean number of PU and DE per observation from Days 214 of cohabitation, indicated that dominant animals engaged in more PU $(M=159.12, S E=18.29)$ than subordinates $(M=7.32, S E=2.86), t(11)=8.31, p<0.001,95 \%$ CI $[111.57,192.03], d=2.40$, and that dominant animals engaged in more DE $(M=32.34, S E=4.10)$ than subordinates $(M=3.86, S E=1.33)$, $t(11)=6.85, p<0.001,95 \% \mathrm{CI}[19.34,37.64], d=1.98$. A similar analysis indicated that DE duration (not depicted in Figure 4) was also greater for dominant animals $(M=70.22 \mathrm{~s}, S E=7.32 \mathrm{~s})$ compared to subordinates $(M=9.54 \mathrm{~s}, S E=2.96 \mathrm{~s}), t(11)=7.14, p<0.001,95 \%$ CI [41.97, 79.39], $d=2.06$. Oneway, repeated measures ANOVAs conducted on difference scores obtained by subtracting the number of PU or DE exhibited by subordinate dyad members from the number exhibited by dominants on Days 2, 4, $6,8,10,12$ and 14 failed to yield significant effects of Day of Cohabitation for both $\mathrm{PU}(F(6,66)=1.14, p$ $=0.350)$ and $\mathrm{DE}(F(6,66)=1.37, p=0.237)$. A similar result was obtained for DE duration $(F(6,66)=$ $1.16, p=0.340)$. Taken together, these results support the argument for behavioral stability.

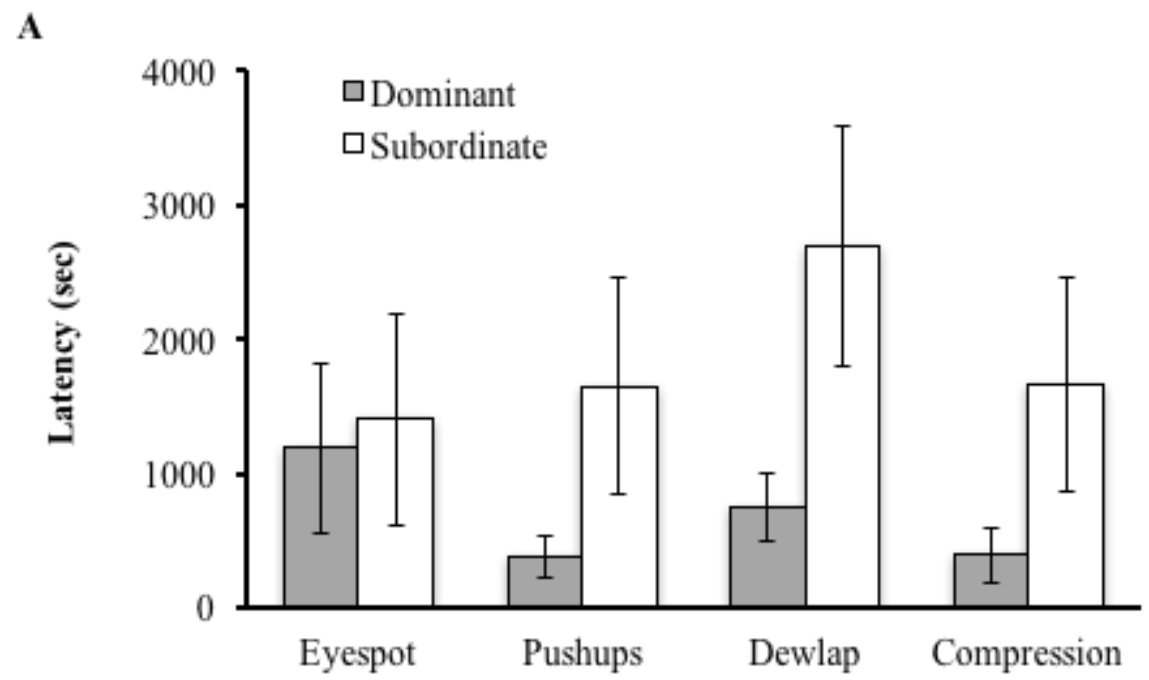

B

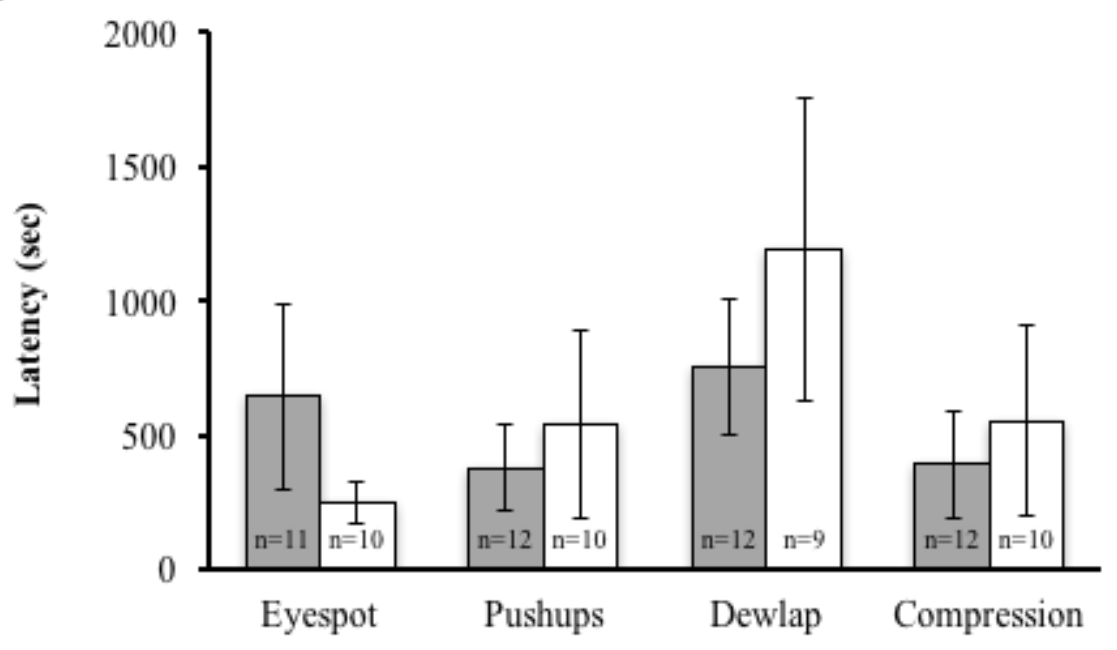

Figure 3. Latencies required for dominant and subordinate animals to exhibit blackening of the postorbital eyespot, pushups, dewlap extensions, and lateral compression of the body during the first two hours of cohabitation. The top panel (A) includes data from all animals in the 12 dyads while the bottom panel (B) includes only the data from animals that exhibited each behavior or characteristic. (Sample sizes are indicated at the base of each bar). Data are presented as means $\pm S E$. 
Social status was also reflected in animals' use of space and access to resources. Dominant animals frequently chased subordinates or displaced them from established cage locations while rarely (two individual occurrences in 83 observations) being chased or displaced themselves (Figure 4c). A t-test for correlated samples confirmed that the mean number of chases and displacements (combined) per observation from Day $2-14$ was higher for dominant animals $(M=3.88, S E=0.79)$ relative to their subordinates $(M=0.02, S E=0.02), t(11)=4.94, p<0.001,95 \% \mathrm{CI}[2.14,5.58], d=1.42)$, and a repeated measures ANOVA indicated that the difference between dominant and subordinate animals was stable from Days $2-14(F(6,66)=1.21, p=0.312)$. In addition, although we did not quantify the behavior exhibited by dominant and subordinate animals during the 30 min courting tests conducted on alternating days from Days $4-14$, observations indicated that males classified as dominant courted more frequently than their subordinate counterparts and/or exhibited aggressive displays or attacks toward subordinate dyad-mates that attempted to court.

Dominant animals were also typically greener than subordinates. There were only three observations (out of 84) where the average body color rating of the subordinate was lower (greener) than the dominant. A t-test for correlated samples confirmed that the average body color per observation from Day 2-14 was greener for dominant animals $(M=1.51, S E=0.09)$ relative to their subordinates $(M=$ $2.47, S E=0.15), t(11)=7.12, p<0.001,95 \%$ CI $[-1.26,-0.66], d=2.05)$, and a repeated measures ANOVA indicated that the that the difference between dominant animals was stable from Days $2-14$ $(F(6,66)=0.623, p=0.711)$.

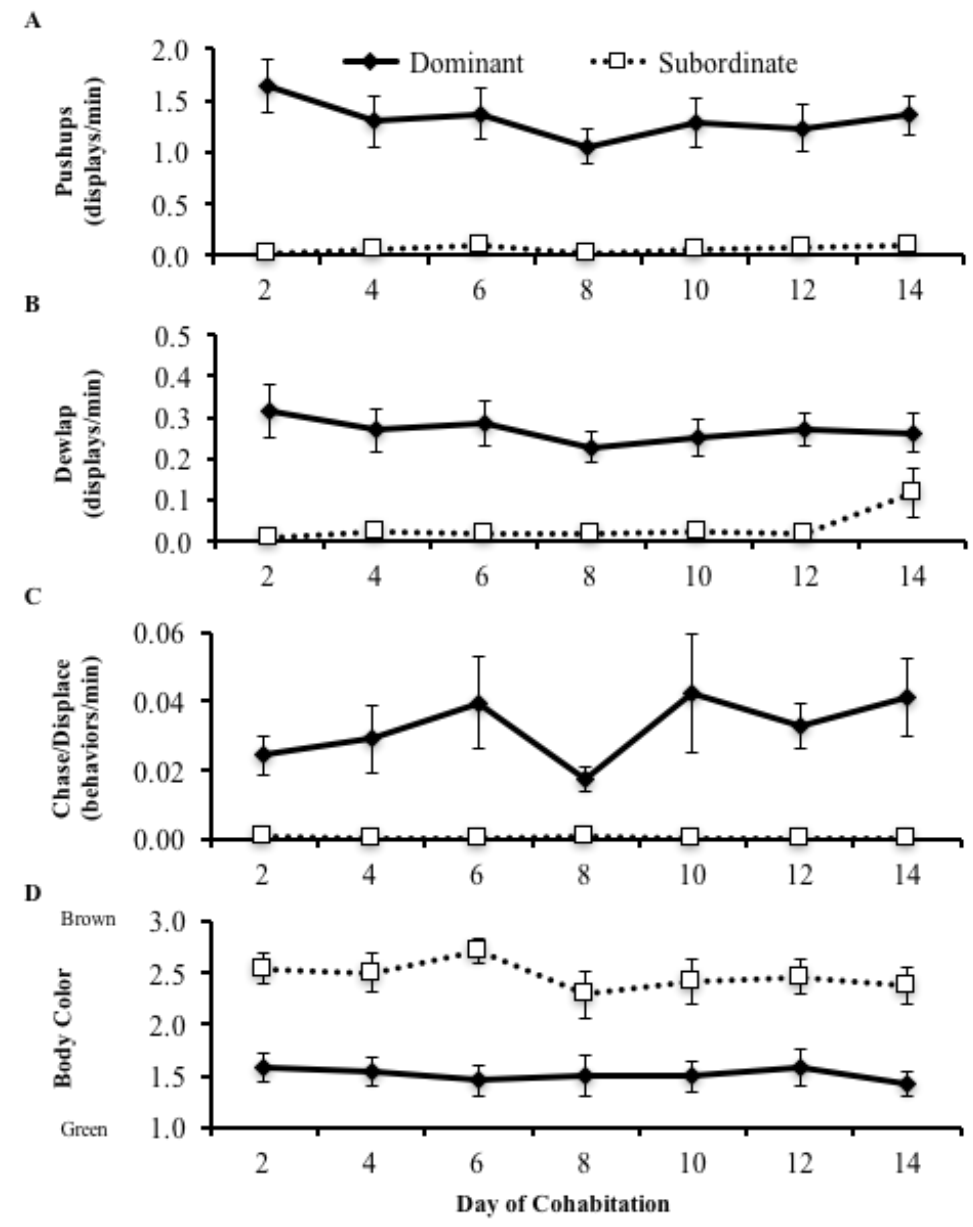

Figure 4. Frequency of Pushups (A), Dewlap Extensions (B), combined Chases and Displacements (C), and mean Body Color (D) exhibited by dominant and subordinate male green anoles during Days $2-14$ of cohabitation. Data are presented as means $\pm S E$. 


\section{Discussion}

This study represents an in-depth analysis of the behavior of male green anole dyads during two weeks of cohabitation. Consistent with previous reports in anoles (Plavicki et al., 2004) and other species (Chase, 1980; Edwards, Issa, \& Herberholz, 2003; Øverli, Harris, \& Winberg, 1999), aggressive behavior was highest at the initiation of interactions (Day 1) and then declined rapidly as stable dominant/subordinate relationships emerged. In addition, the asymmetry in display behavior between dominant and subordinate animals that existed from Days $2-14$ of cohabitation reliably emerged during the first two hours of dyadic interaction.

The finding that measures of display intensity/persistence (PU and DE frequency and DE duration) were higher in dominant animals during the first two hours of cohabitation is consistent with several other reports regarding the winners of dyadic interactions in this species (Korzan et al., 2007; Ling, Summers, Renner, \& Watt, 2010; Meyer, Keifer, Korzan, \& Summers, 2004; Plavicki et al., 2004; Wilczynski et al., 2015). This finding is also in agreement with the conceptualization of aggressive displays as honest signals. Honest signals are signals that are reliably correlated with some aspect of the signaler that the receiver benefits from having information about such as fighting ability or territory holding capacity (Searcy \& Nowicki, 2005; Zahavi, 1977). Although the relationship between the frequency and/or duration of aggressive displays and fighting ability or territory holding capacity have not been systematically examined in green anoles, Simon (2011) found that higher male DE and bobbing (similar to PU) rates in a population of brown anoles (A. sagrei) predicted successful territorial defense. In addition, Perry, LeVering, Girard, and Garland (2004) found that amongst male A. cristatellus, winners of staged aggressive interactions exhibited more assertion displays before capture in the field and had higher levels of locomotor endurance (measured on a treadmill) than losers of interactions. Additionally, they reported a significant positive correlation between display rate in the field and locomotor endurance. Taken together these results support the hypothesis that aggression-related displays may be an honest signal of relevant aggression-related variables in anoles.

The finding that the latencies to exhibit aggressive displays and blacken the postorbital eyespot at the beginning of cohabitation did not differ between dominant and subordinate animals was somewhat surprising. Several authors have reported that shorter latencies to eyespot blackening reliably distinguish winners from losers of aggressive interactions (Larson \& Summers, 2001; Plavicki et al., 2004; Summers \& Greenberg, 1994). In addition, it has been demonstrated that altering eyespot color with black or green paint can influence mirror-elicited aggression (Korzan, Summers, Ronan, \& Summers, 2000) and dyadic aggressive interactions (Korzan, Forster, Watt, \& Summers, 2006; Korzan, Summers, \& Summers, 2002) such that males viewing an opponent with a blackened eyespot are less aggressive. Shorter latencies to express other aggressive behaviors have also been reported for the winners of green anole aggressive contests (Korzan, Øverli et al., 2006) and numerous studies suggest that individuals with a proactive stress coping style characterized by rapid physiological and behavioral responses to both social and nonsocial stressors are typically more aggressive and/or more likely to win than those who adopt a slower, reactive, approach (Dahlbom, Lagman, Lundstedt-Enkel, Sundstrom, \& Winberg, 2011; David, Auclair, \& Cézilly, 2011; Koolhaas, de Boer, Coppens, \& Buwalda, 2010; Koolhaas et al., 1999; Korzan, Øverli et al., 2006; Øverli et al., 2004; Sundstrom, 2004).

Although our findings differ from those above, they are in agreement with previous observations in our laboratory (Zaman \& Farrell, 2007) and those of Wilczynski et al. (2015) who found the amount of display behavior exhibited by male green anoles to be a more robust predictor of contest outcome than behavioral and eyespot blackening latencies. Although numerous factors could explain the discrepancies between the latency data reported here and those of others, one possibility is that housing conditions may have played a role. Unlike most other researchers, but similar to Wilczynski et al. (2015), we housed our male anoles with females prior to inclusion in this study. Perhaps the presumably higher value of these pre-contest territories influenced initial reactivity when later faced with an opponent. Other methodological factors, such as our placement of the perch in the testing enclosure immediately following 
removal of the wall separating dyad members at the beginning of the cohabitation period may also have influenced our findings.

While the latency to eyespot blackening, an indicator of increased levels of circulating catecholamines (Goldman \& Hadley, 1969), did not differ between dominants and subordinates, body color rapidly diverged during the first two hours of cohabitation such that subordinate animals were significantly browner than dominants. These changes are mediated by stress-related hormones: melanocyte-stimulating hormone (MSH), which along with adrenocorticotropic hormone (ACTH) is a derivative of pro-opiomelanocortin (POMC), causes browning of body skin, and catecholamines can modulate skin color via $\alpha 2$ - and $\beta 2$-adrenergic receptors rendering the skin greener and browner respectively (Goldman \& Hadley, 1970; Vaughan \& Greenberg, 1987). Browner body color is often reported as characteristic of both long-term subordinate animals (Greenberg et al., 1984; Greenberg \& Crews, 1990; Plavicki et al., 2004) and the losers of acute agonistic interaction (Summers \& Greenberg, 1994; Wilczynski et al., 2015), and can co-occur with increased titers of corticosterone during long-term cohabitation (Greenberg et al., 1984). Our observations corroborate previous reports that this chromatic marker of endocrine state changes rapidly during aggressive interactions and that dominant animals are typically greener than subordinates.

Our data also suggest that measures of display intensity/persistence and body color can also be used to reliably indicate social status at the level of individual dyads. Whereas no individual measure was able to correctly distinguish the eventual dominant animal from the subordinate in all 12 dyads, the dominant animal in 11 of 12 dyads had higher frequencies of PU and DE after two hours of cohabitation. Similarly, the mean body color of dominants was greener than their opponent in 10 of the 12 dyads. If we employed a compound rule to distinguish dominants from subordinates with the requirement that the dominant animal meet two out of three of these criteria (more PU, more DE and greener mean body color) 12 of 12, 11 of 12, and 10 of 12 dominant animals are correctly identified after 120,90 and 60 min of interaction respectively. The percentage of correct identifications declines to $66.67 \%$ ( 8 of 12 dyads) following only $30 \mathrm{~min}$ of interaction. Compound rules of this type could be useful for those trying to operationally distinguish between dominant and subordinate animals when using social status as either a dependent measure or a quasi-independent variable across which comparisons are made.

The data collected from Days 2 - 14 of cohabitation allowed us to provide the most in-depth description and analysis of the display behavior exhibited by male green anoles during long-term cohabitation to date. Detailed longitudinal data documenting the behavior of green anoles during longterm, dyadic cohabitation are rarely reported in the literature, and when they are (e.g., Plavicki et al., 2004), they have been limited to measures of body color and perch site selection. Detailed data regarding aggression-related motor displays are likely lacking, in part because the use of brief behavioral observations to quantify social status during long-term cohabitation precludes collecting representative samples of these behaviors. The use of two-hour video observations allowed us to observe multiple aggressive displays for all of our dyads on every day of sampling and quantify common displays such as PU and DE frequency, thereby allowing us to present data demonstrating the consistency with, and the degree to which, dominant animals out-display their opponents. The findings from this analysis (Figure 4) indicate that the differences in display intensity/persistence seen during the first two hours of cohabitation persist during long-term dyadic housing and are stable for two weeks.

The collection of longer video records during extended cohabitation also allowed us to quantify variables related to the dominance of space use (chases and displacements) in ways that do not require the researcher to determine a priori which areas of the habitat are "preferred." While many of the dominant animals we observed appeared to exhibit preferences for the highest perch location, as is commonly reported, both dominant and subordinate animals often changed their position within the habitat. Because observers could easily change the direction of video playback during behavioral coding, we were able to register a displacement whenever an animal vacated a position it had occupied for a minimum of $30 \mathrm{~s}$ prior to a display or approach by the displacing animal. The only assumption to this operationalization is that animals prefer to occupy the positions they choose to inhabit within the environment. Given that 
animals may choose to change their position within the environment to regulate physiological variables such as body temperature, this distinction is of potential importance.

Examination of the video record also revealed that typical summary data do not fully capture the dynamics of body color regulation during long-term cohabitation. While the body color data we report from Days 2-14 of cohabitation (Figure 4d: the mean of measures taken at the beginning and end of the observation) might suggest that body color became static, this was often not the case. During both twohour dyadic observations, and following the introduction of a female into the enclosure for 30 minutes on alternating days from Day 2 - 14 of cohabitation, dominant and subordinate animals frequently changed color. Dominant animals often turned brown for brief periods of time coinciding with bouts of courting or aggressive display. Subordinate animals also sometimes became green, but typically became brown again shortly thereafter.

Our findings regarding the stability of social status from Days 2-14 of cohabitation are generally consistent with reports from other researchers (Greenberg \& Crews, 1990; Plavicki et al., 2004; Summers \& Greenberg, 1994) in that aggression was reduced relative to the initial phase of interaction, dominant animals were typically greener than subordinates, and dominants were able to control the use of space and access to mates. These findings are also consistent with several reports of opponent recognition and the possibility of a "dear enemy" effect (Fisher, 1954) in anoles whereby animals remember the identity of previous opponents, reduce their emissions of aggressive displays, and maintain stable social status relationships when confronted with a familiar real or simulated (video) opponent (Fisher, 1954; Forster et al., 2005; Larson \& Summers, 2001; Qualls \& Jaeger, 1991; Yang et al., 2001). Importantly, however, because we examined animals during continuous cohabitation we cannot assess the memory component of this proposition.

Taken together, our findings demonstrate that asymmetries in aggressive display behavior and body color between members of green anole dyads after the first day of cohabitation persist in a stable fashion for up to two weeks. Animals operationally defined as dominant on Day 2 of cohabitation consistently displayed more PU and DE and were greener than their subordinate counterparts through Day 14. Our data also indicate that these asymmetries emerge quite rapidly. Measures of display intensity/persistence and body color during the first two hours of cohabitation were reliable indicators of future status both on average, and at the level of individual dyads.

\section{Acknowledgements}

Support for this research was provided by the Franklin \& Marshall College Hackman Summer Scholars Program and Faculty Research and Professional Development Fund. We wish to thank Dr. Walter Wilczynski for his helpful comments on an earlier version of this manuscript and Ashley Bolig for her assistance with behavioral coding.

\section{References}

Becker, E. A., \& Marler, C. A. (2015). Postcontest blockade of dopamine receptors inhibits development of the winner effect in the California mouse (Peromyscus californicus). Behavioral Neuroscience, 129, $205-213$.

Camras, L. A. (1977). Facial expressions used by children in a conflict situation. Child Development, 48, 14311435.

Chase, I. D. (1980). Social process and hierarchy formation in small groups: A comparative perspective. American Sociological Review, 45, 905-924.

Crews, D. (1975). Inter- and intraindividual variation in display patterns in the lizard, Anolis carolinensis. Herpetologica, 31, 37-47.

Crews, D., Traina, V., Wetzel, F. T., \& Muller, C. (1978). Hormonal control of male reproductive behavior in the lizard, Anolis carolinensis: Role of testosterone, dihydrotestosterone, and estradiol. Endocrinology, 103, 1814-1821.

Dahlbom, S. J., Lagman, D., Lundstedt-Enkel, K., Sundstrom, L. F., \& Winberg, S. (2011). Boldness predicts social status in zebrafish (Danio rerio). PLoS ONE, 6, e23565. 
David, M., Auclair, Y., \& Cézilly, F. (2011). Personality predicts social dominance in female zebra finches, Taeniopygia guttata, in a feeding context. Animal Behaviour, 81, 219-224.

de Almeida, R. M., Cabral, J. C., \& Narvaes, R. (2015). Behavioural, hormonal and neurobiological mechanisms of aggressive behaviour in human and nonhuman primates. Physiology \& Behavior, 143, 121-135.

Edwards, D. H., Issa, F. A., \& Herberholz, J. (2003). The neural basis of dominance hierarchy formation in crayfish. Microscopy Research and Technique, 60, 369-376.

Fisher, J. (1954). Evolution and bird sociality. In J. Huxley, A. C. Hardy \& E. B. Ford (Eds.), Evolution as a process (pp. 71 - 83). London, England: Allen \& Unwin.

Forster, G. L., Watt, M. J., Korzan, W. J., Renner, K. J., \& Summers, C. H. (2005). Opponent recognition in male green anoles, Anolis carolinensis. Animal Behaviour, 69, 733-740.

Fuxjager, M. J., Forbes-Lorman, R. M., Coss, D. J., Auger, C. J., Auger, A. P., Marler, C. A., \& McEwen, B. S. (2010). Winning territorial disputes selectively enhances androgen sensitivity in neural pathways related to motivation and social aggression. Proceedings of the National Academy of Sciences of the United States of America, 107, 12393-12398.

Goldman, J. M., \& Hadley, M. E. (1969). In vitro demonstration of adrenergic receptors controling responses of the lizard, Anolis carolinensis. Journal of Pharmacology and Experimental Therapeutics, 166, 1-7.

Goldman, J. M., \& Hadley, M. E. (1970). Evidence for separate receptors for melanophore stimulating hormone and catecholamine regulation of cyclic AMP in the control of melanophore responses. British Journal of Pharmacology, 39, 160-166.

Goldstein, M. H., \& Brodsky, P. (2006). EventCoder (Version 1.0b11). Cornell University.

Grant, E. C. (1969). Human facial expression. Man, 4, 525-692.

Greenberg, N., Chen, T., \& Crews, D. (1984). Social status, gonadal state, and the adrenal stress response in the lizard, Anolis carolinensis. Hormones and Behavior, 18, 1-11.

Greenberg, N., \& Crews, D. (1983). Physiological ethology of aggression in amphibians and reptiles. In B. Svare (Ed.), Hormones and aggressive behavior (pp. 469 - 506). Boston, MA: Springer.

Greenberg, N., \& Crews, D. (1990). Endocrine and behavioral responses to aggression and social dominance in the green anole lizard, Anolis carolinensis. General and Comparative Endocrinology, 77, 246-255.

Haller, J. (2014). The glucocorticoid/aggression relationship in animals and humans: An analysis sensitive to behavioral characteristics, glucocorticoid secretion patterns, and neural mechanisms. In K. A. Miczek \& A. Meyer-Lindenberg (Eds.), Neuroscience of aggression (Vol. 17, pp. 73 - 109). Berlin, Germany: Springer.

Hsu, Y., Earley, R. L., \& Wolf, L. L. (2006). Modulation of aggressive behaviour by fighting experience: Mechanisms and contest outcomes. Biological Reviews of the Cambrige Philosophical Society, 81, 33-74.

Jenssen, T. A., Greenberg, N., \& Hovde, K. A. (1995). Behavioural profile of free-ranging male lizards, Anolis carolinensis, across breeding and post-breeding seasons. Herpetological Monographs, 9, 41-62.

Jenssen, T. A., \& Nunez, S. C. (1998). Spatial and breeding relationships of the lizard, Anolis carolinensis: Evidence of intrasexual selection. Behaviour, 135, 981-1003.

Koolhaas, J. M., de Boer, S. F., Coppens, C. M., \& Buwalda, B. (2010). Neuroendocrinology of coping styles: Towards understanding the biology of individual variation. Frontiers in Neuroendocrinology, 31, $307-321$.

Koolhaas, J. M., Korte, S. M., De Boer, S. F., Van Der Vegt, B. J., Van Reenen, C. G., Hopster, H., . . . Blokhuis, H. J. (1999). Coping styles in animals: Current status in behavior and stress-physiology. Neuroscience \& Biobehavioral Reviews, 23, 925-935.

Korzan, W. J., Forster, G. L., Watt, M. J., \& Summers, C. H. (2006). Dopaminergic activity modulation via aggression, status, and a visual social signal. Behavioral Neuroscience, 120, 93-102.

Korzan, W. J., Höglund, E., Watt, M. J., Forster, G. L., Øverli, Ø., Lukkes, J. L., \& Summers, C. H. (2007). Memory of opponents is more potent than visual sign stimuli after social hierarchy has been established. Behavioural Brain Research, 183, 31-42.

Korzan, W. J., Øverli, Ø., \& Summers, C. H. (2006). Future social rank: Forecasting status in the green anole (Anolis carolinensis). Acta Ethologica, 9, 48-57.

Korzan, W. J., \& Summers, C. H. (2007). Behavioral diversity and neurochemical plasticity: Selection of stress coping strategies that define social status. Brain, Behavior and Evolution, 70, 257-266.

Korzan, W. J., Summers, T. R., Ronan, P. J., \& Summers, C. H. (2000). Visible sympathetic activity as a social signal in Anolis carolinensis: Changes in aggression and plasma catecholamines. Hormones and Behavior, 38, 193-199.

Korzan, W. J., Summers, T. R., \& Summers, C. H. (2002). Manipulation of visual sympathetic sign stimulus modifies social status and plasma catecholamines. General and Comparative Endocrinology, 128, 153161. 
Larson, E. T., \& Summers, C. H. (2001). Serotonin reverses dominant social status. Behavioural Brain Research, $121,95-102$.

Licht, P. (1971). Regulation of the annual testis cycle by photoperiod and temperature in the lizard anolis carolinensis. Ecology, 52, 240-252.

Ling, T. J., Summers, C. H., Renner, K. J., \& Watt, M. J. (2010). Opponent recognition and social status differentiate rapid neuroendocrine responses to social challenge. Physiology \& Behavior, 99, 571-578.

Meyer, W. N., Keifer, J., Korzan, W. J., \& Summers, C. H. (2004). Social stress and corticosterone regionally upregulate limbic N-methyl-D-aspartatereceptor (NR) subunit type $\mathrm{NR}(2 \mathrm{~A})$ and $\mathrm{NR}(2 \mathrm{~B})$ in the lizard Anolis carolinensis. Neuroscience, 128, 675-684.

Øverli, Ø., Harris, C. A., \& Winberg, S. (1999). Short-term effects of fights for social dominance and the establishment of dominant-subordinate relationships on brain monoamines and cortisol in rainbow trout. Brain, Behavior and Evolution, 54, 263-275.

Øverli, Ø., Korzan, W. J., Höglund, E., Winberg, S., Bollig, H., Watt, M., . . Summers, C. H. (2004). Stress coping style predicts aggression and social dominance in rainbow trout. Hormones and Behavior, 45, $235-241$.

Oyegbile, T. O., \& Marler, C. A. (2005). Winning fights elevates testosterone levels in California mice and enhances future ability to win fights. Hormones and Behavior, 48, 259-267.

Perry, G., LeVering, K., Girard, I., \& Garland, T. (2004). Locomotor performance and social dominance in male Anolis cristatellus. Animal Behaviour, 67, 37-47.

Plavicki, J., Yang, E. J., \& Wilczynski, W. (2004). Dominance status predicts response to nonsocial forced movement stress in the green anole lizard (Anolis carolinensis). Physiology and Behavior, 80, 547-555.

Qualls, C. P., \& Jaeger, R. G. (1991). Dear enemy recognition in Anolis carolinenis. Journal of Herpetology, 25, 361-363.

Riechert, S. E. (1998). Game theory and animal contests. In L. A. Dugatkin \& H. K. Reeve (Eds.), Game theory and animal behavior (pp. 64 - 93). New York, NY: Oxford University Press.

Ruby, D. E. (1984). Male breeding success and differential access to females in Anolis carolinensis. Herpetologica, 40, 272-280.

Ryan, M. J., \& Wilczynski, W. (2011). Introduction to animal behavior: An integrative approach. Cold Springs Harbor, NY: Cold Springs Harbor Press.

Searcy, W. A., \& Nowicki, S. (2005). The evolution of animal communication: Reliability and deception in signaling systems. Princeton, NJ: Princeton University Press.

Simon, V. B. (2011). Communication signal rates predict interaction outcome in the brown anole lizard, Anolis sagrei. Copeia, 2011, 38-45.

Soma, K. K., Scotti, M. A., Newman, A. E., Charlier, T. D., \& Demas, G. E. (2008). Novel mechanisms for neuroendocrine regulation of aggression. Frontiers in Neuroendocrinology, 29, 476-489.

Summers, C. H. (2001). Mechanisms for quick and variable responses. Brain, Behavior and Evolution, 57, $283-292$.

Summers, C. H., Forster, G. L., Korzan, W. J., Watt, M. J., Larson, E. T., Øverli, Ø., . . Greenberg, N. (2005). Dynamics and mechanics of social rank reversal. Journal of Comparative Physiology A, 191, 241-252.

Summers, C. H., \& Greenberg, N. (1994). Somatic correlates of adrenergic activity during aggression in the lizard, Anolis carolinensis. Hormones and Behavior, 28, 29-40.

Summers, C. H., \& Greenberg, N. (1995). Activation of central biogenic amines following aggressive interaction in male lizards, Anolis carolinensis. Brain, Behavior and Evolution, 45, 339-349.

Summers, C. H., \& Winberg, S. (2006). Interactions between the neural regulation of stress and aggression. Journal of Experimental Biology, 209, 4581-4589.

Sundstrom, L. F. (2004). Hatchery selection promotes boldness in newly hatched brown trout (Salmo trutta): Implications for dominance. Behavioral Ecology, 15, 192-198.

Vaughan, G. L., \& Greenberg, N. (1987). Propanolol, a beta-adrenergic antagonist, retards response to MSH in skin of Anolis carolinensis. Physiology \& Behavior, 40, 555-558.

Wallen, K. (2005). Hormonal influences on sexually differentiated behavior in nonhuman primates. Frontiers in Neuroendocrinolology, 26, 7-26.

Watt, M. J., Forster, G. L., Korzan, W. J., Renner, K. J., \& Summers, C. H. (2007). Rapid neuroendocrine responses evoked at the onset of social challenge. Physiology \& Behavior, 90, 567-575.

Wilczynski, W., Salem, S. J., Ezeoke, Chisom B., \& Black, M. P. (2015). Behavioural persistence during an agonistic encounter differentiates winners from losers in green anole lizards. Behaviour, 152, 563-591.

Wingfield, J. C., \& Wada, M. (1989). Changes in plasma levels of testosterone during male-male interactions in the song sparrow, Melospiza melodia: Time course and specificity of response. Journal of Comparative Physiology A, 166, 189-194. 
Wittig, R. M., Crockford, C., Weltring, A., Deschner, T., \& Zuberbuhler, K. (2015). Single aggressive interactions increase urinary glucocorticoid levels in wild male chimpanzees. PLOS ONE, 10, e0118695.

Yang, E.-J., Phelps, S. M., Crews, D., \& Wilczynski, W. (2001). The effects of social experience on aggressive behavior in the green anole lizard (Anolis carolinensis). Ethology, 107, 777-793.

Yang, E.-J., \& Wilczynski, W. (2003). Interaction effects of corticosterone and experience on aggressive behavior in the green anole lizard. Hormones and Behavior, 44, 281-292.

Zahavi, A. (1977). Reliability in communication systems and the evolution of altruism. In B. Stonehouse \& C. M. Perrins (Eds.), Evolutionary ecology (pp. 253 - 259). London, England: Macmillan.

Zaman, S., \& Farrell, W. J. (2007). Quantification of the outcome of aggressive interactions between male green anole lizards (Anolis carolinensis). Poster presented at the annual meeting of the Society for Behavioral Neuroendocrinology, Pacific Grove, CA. 